\title{
KASAYSAYAN, SINING, LIPUNAN: ANG POLITIKA NG PANITIKAN SA MAKABAGONG PANAHON
}

\author{
E. San Juan, Jr. \\ Philippine Cultural Studies Center \\ Connecticut, USA \\ philcsc@gmail.com
}

\begin{abstract}
Abstrakto
Sa realidad ng ating sitwasyong neokolonyal, isinasakatuparan ng araling panliteratura ang minanang tungkuling ginagampanan nito na gawing masunuring sabjek ang indibidwal na may kakayahang mangatwiran. Sinusugpo ng normatibong ideolohiya ang pagsusuri, at tuloy ikinukubli ang karanasan ng eksploytasyon at tunggalian ng mga uri. Sinusupil ang mismong konsepto ng ideolohiya, itinatakwil ang mga konteksto't praktikang panginstitusyonal na siyang yumayari dito. Gamit ang historistiko't sintomatikong pagbasa ng nobela ni Lualhati Bautista bilang halimbawa, tinatalakay dito ang pagkakaiba ng ideolohiya ng teksto sa diwa ng awtor dahil sa maraming namamagitang puwersa tulad ng alyenasyon at reipikasyon. Sa bisa ng sari-saring mekanismong pang-interpelasyon, hinuhubog ng teksto ang mga nagbabasa upang maging sabjek na nagsusumikap lutasin sa guniguni ang umiiral na tahasang kontradiksyong panlipunan. Sa gitna ng konkretong awdiyens na taglay ang sapin-saping pagkakaiba sa kultura at wika, ang pagpili ng wikang kasangkapan sa pagbuo ng likhang-sining ay maselang desisyon. Ilugar ito sa proseso ng paghabi ng mga kahulugan ng teksto bilang masalimuot at dinamikong repleksyon ng isang tiyak na conjuncture sa daloy ng kasaysayan ng bansang Pilipinas.
\end{abstract}

Given the reality of our neocolonial situation, the discipline of literary study fulfills its received pedagogical function of converting reason-bearing individuals into obedient subjects. This normative ideology represses criticism, concealing the lived experience of exploitation and class conficts. The concept of ideology itself is suppressed, replaced with accepted terms and approved habits of interpretation. Meanings of art-works are identified with the single author's identity, thus ignoring or excluding their enabling contexts and institutional practices. Using a symptomatic, historicist reading of Lualhati Bautista's novel as an example, this essay argues that the ideology of texts differs from that of the author owing to diverse mediating factors. Current forces of alienation and reification intervene. Through their own mechanisms of interpellation, texts transform readers into subjects who attempt to imaginarily resolve real social contradictions. Given our multilingual and multiethnic audience, the choice of language for literary expression becomes crucial in the 
process of articulating textual meanings as multifaceted, dynamic reflections of specific conjunctures in Philippine history.

\section{Keywords}

Awtor, Diskurso, Ideolohiya, Indibidwal, Kahulugan, Kontradiksyon, Sabjek

\section{About the Author}

E. San Juan, Emeritus Professor of English, Comparative Literature and Ethnic Studies, Washington State University, was recently fellow of the WEB Du Bois Institute, Harvard University, and of the Harry Ransom Center, University of Texas, Austin. He is currently professorial lecturer of Cultural Studies, Polytechnic University of the Philippines. His recent books are Ulikba (UST Publishing House), Kundiman sa Gitna ng Karimlan (UP Press), Ambil, and the forthcoming Between Empire and Insurgency (UP Press) and Lupang Hinirang, Lupang Tinubuan (De La Salle University Press).

\section{Editor's Note}

An early version of this paper was delivered as a plenary lecture in the Kritika KulturaAteneo Center for English Language Teaching National Conference-Workshop 2014: Literature, the Region, and the World in K-12; the conference was held on Feb. 15, 2014 at the Ateneo de Manila University. 
1. Sa unang malas, umaayaw na o natatabangan ang marami sa pagkasulyap sa salitang "ideolohiya." Ano ba ito, propaganda o chika tungkol sa politika na hindi bagay sa okasyong itong pagsunod sa binagong K-12 curriculum. Kung inyong nabasa ang Batas at Memo ni Dir. Licuanan, nais daw hasain ang estudyante sa kritikal at malikhaing pag-iisip upang itransporma ang sarili at kapaligiran. Naku, bigating layunin ito. Idiniin ding kailangan daw iakma o iayon ang turo't aralin sa global istandard.

2. Isa sa required reading sa mga kolehiyo sa Europa \& mga bansa sa Aprika at Amerika ang The German Ideology nina Marx at Engels. Tiyak na alam ng lahat na bawal ang komunistang lathalai't usapan sa mga klasrum, lalo na sa panahon ng "Cold War." Tapos na ito sa buong mundo, pero patuloy pa rin tayo sa mentalidad ng pagbabawal. Kung sa bagay, ngayon ang panahon ng "total surveillance," at hanggang ngayon, ang premyadong pelikulang "Orapronobis" ni Lino Brocka ay hindi pinahihintulutang maipalabas sa publiko.

Sa ano't anuman, ang "ideolohiya” ay salitang laganap na sa iba't ibang sangay ng aralin sa humanidades at siyensiyang sosyal. Hindi na dapat pagtalunan kung bakit mahalagang siyasatin ang kaugnayan nito sa pagtuturo. Halimbawa na nga ang inyong pagkataka o pagkamangha, kung nangyari nga, sa paglitaw nito sa programa.

3. Isa pang halimbawa: Malimit nating pasalamatan ang mga burokratang opisyal sa pagtustos sa pagpupulong tulad nito. Sa katunayan, ang dapat pasalamatan ay mga manggagawa, magbubukid at empleyadong tulad ninyo na siyang yumayari ng kayamanan ng bansa. Ang lakas-paggawa ng karaniwang mamamayan ang nagbubuwis ng halaga upang mangyari ang miting na ito, kaya sa kanila ang pasasalamat natin - hindi sa mga politiko at kanilang "pork-barrel" na nakaw sa pagod ng mga anak-pawis, bukal ng anumang kabihasnan.

4. Sa gayon, ang ideolohiya ay hindi lamang pumapatnubay na ideya o paniniwala kundi kilos, gawi, praktika. Ito ay mga institusyong siyang gumagawa ng sabjek sa bawat indibidwal. Ang sabjek ang siyang aktor na kinikilala, nagpapasiya, kumikilos, may pananagutan.

Makikita sa relasyon ng titser at estudyante: "Hoy, makinig kayo!" giit ng guro. Awtoridad ang titser, kinatawan ng Estado at siyang nagdedeposito ng kaalaman sa basyong utak ng mga kabataan. Ito ang "banking method" ng edukasyon na tinuligsa ni Paulo Freire. Galing ito sa mahabang karanasan natin sa disiplinang pedagohikal ng kolonyalismong Espanyol at Amerikano. 
Sa pagsakop ng ekonomiyang nakaangkla sa "exchange value" ng trabaho, wikang Ingles ang instrumento sa paghulma ng sabjek ng malayang pamilihan ipagbili ang lakas-paggawa kung may bibili. Bagamat ang hangarin ng makabagong sistema ay indibidwal na may nagsasariling katwiran ("autonomous rational mind," naisaad ni Immanuel Kant), kaiba ang resulta: ginagawang masunuring sabjek ang indibidwal sa posisyon niya sa istruktura ng lipunan.

5. Ang lipunan ay katumbas sa ugnayan o relasyon ng mga sabjek. Hindi ito kumpol lamang ng hiwa-hiwalay na inbidwal. Bawat identidad/kaakuhan halimbawa, sabjek bilang awtor, mambabasa, guro, at iba pa - ay nabubuo lamang sa loob ng ugnayang panlipunan. Samakatuwid, ang sabjek ay produkto ng pagkilala, pagtawag, interpelasyon ng diskurso, praktika, institusyon.

Hindi kaakuhan/identidad ng awtor ang pinagmumulan ng kahulugan ng akda. Iyon ay bunga ng diskurso, ng tekstong binubuo ng magkasalungatang puwersang nagsusulong sa kasayaysan. Ang indibidwal ay ginagawang sabjek ng wika sa diskursong gamit ng mga institusyong ideolohikal. Makapangyarihan ang asignatura sa panitikang Filipino sa paglalantad ng sitwasyon kung saan ang wikang Ingles ay dominante pa rin, tanda ng poder ng modernisadong oligarkiya, na bunyag nga sa paggamit ng "mother tongue" sa unang baytang ng iskwela. Pahiwatig na sa kompitensiya ng wika, nananaig pa rin ang poder ng bangko't korporasyong global ng Estados Unidos, Europa, Hapon, at sirkulo ng industriyalisadong bansa. Ang poder ay naisakatawan sa wikang Ingles, o sa mga "englishes," na bumubuhay sa gahum o hegemonya nito sa buong daigdig.

Sa pamamagitan ng mga institusyon at operasyong praktikal nito, kinikilalang sabjek ang sinuman upang makaganap ng takdang tungkulin sa isang tiyak na lugar sa kasaysayan ng lipunan. Gayundin ang awtor: batay sa institusyonalisadong praktika, ang identidad ng awtor at gawa niya ay nakasalig sa pagtawag at pagkilala sa kanya ng namamayaning pananaw - ang normatibong paniniwalang operasyonal sa gawi, batas, at iba pa kung saan nakasandig ang kapangyarihan ng dominanteng uri sa hinating lipunan.

6. Walang sitwasyong permanente sa kasaysayan. Lapatan natin ng historikal na panimbang ang pabago-bagong pagtingin sa awtor at akdang itinuturo natin.

Namihasa tayong ipalagay na ang isang akda ay bunga ng henyo o talino ng awtor. Iba noong sinaunang panahon: ang awtor ng epiko, korido, pasyon, at iba pa ay kabilang sa pangkat na naglilingkod sa lider ng tribu, ng simbahan o aristokrasya (tulad ni Balagtas). Ginagabayan sila ng kombensiyon, determinadong kodigo, at panuntunang institusyonal. 
Nag-iba ito paglipas ng Renaissance; tuluyang humiwalay ang artisano't naging negosyante ng kanyang dunong sa sinumang bibili nito. Malaya na siya sa malas, pero alipin naman ng pamilihan. Ganito pa rin ang sitwasyon ng awtor o sinumang intelektwal (guro, peryodista, at iba pa) na walang pag-aari ng kailangang kagamitan upang mabuhay.

Noong ika-19 siglo, umaklas ang mga artista laban sa burgesyang orden ng kapitalismong industriyal. Batay sa romantikong pananaw, ang awtentikong galing ng manunulat ay tiwalag sa burgesyang lipunan at indibidwalistikong pamantayan nito. Itinuring na doon nagmumula ang kahulugan at katuturan ng akda. Kalaunan, pinatingkad ito ng ideolohiya ng sistemang kapitalismo, bagamat ang normatibong mapang-angkin ay tinuligsa nina Flaubert, Zola, Dostoevsky, Gorki, Dreiser, Hemingway, at iba pa.

Ang rebelyon nina Villa, Abadilla, Amado Hernanez, at mga modernistang sumunod ay sintomas ng krisis ng sitwasyon ng petiburgesyang intelektwal sa neokolonyang predikamentong tumitingkad at lumalala ngayon.

7. Sa pagsipat at pagkilatis ng diskurso, mapapansin na impluwensiyal pa rin ang klasikong pamantayan nina Regalado, Balmaseda, Panganiban: ang "dulce et utile" ni Horace pinatining ng romantikong ideyalismo. Nitong dekada 70 at 80 pumasok ang formalismo, feminismo, istrakturalismo't iba pang tatak postmodernistang perspektibo, sa neoliberalismong bandila ng "end of ideology" o tandisang tagumpay ng kapitalismo pagbulusok ng Unyon Sobyet at alternatibong radikal.

Tatlong lapit ang resulta. Una, ang didaktikong motibasyon: birtud ng literatura ang pumukaw ng diwa o damdamin at magbigay ng aral na mapapakinabangan. Masisinag ang utilitaryanismong ugat sa dokumentong nabanggit. Pangalawa, ang empirisistikong hilig na sa reyalistikong pamamaraan nailalahad ng akda ang katotohanan, tiwalag sa anumang paniniwalang moral o etikal ng awtor. Pangatlo, aliw ang dulot ng ayos o porma ng sining; kariktan o kagandahan ang mahalagang katangian nito para sa esklusibo't sopistikadong sensibilidad.

Matatarok na sintomas ito ng kontradiksiyon ng mga grupo sa lipunan: ang awtoridad ng mga naghaharing uring may mala-pyudal na pangitain, laban sa komprador-burokratang saray na tutok sa pamantayang global at siyentipikong pamantayan. May panggitnang oryentasyon din.

Ngunit hinahanap pa, dili kaya'y hinihitay pa, ang pagtinging sumasalungat ng mga nakararami, ang interpretasyong nagsisiwalat ng pagtatagisan ng samutsaring sektor ng lipunan at pagkakabuhol ng mga ito. Sa madaling salita, ang katotohanan ng kasalukuyan sitwasyon at kalakaran ng tunggalian ng mga uri.

8. Batay sa kuro-kurong naisaad, atupagin natin ang pangunahing tanong: Sa tawag ng nobela ni Bautista, anong sabjek ang nabubuo mula sa karanasan ng pagbasa? Upang matugon ito, marahil dapat usisain muna ang ating sitwasyon at kinalalagyan. Anong kondisyon ng ating lipunan sa ngayon? Bilang guro at 
estudyante, saan tayo nakapwesto sa kasalukuyang krisis ng kapitalismong global at alitang rehiyonal? Ano ang tungkulin natin sa institusyong inutusan ng Estado na hubugin ang isip at damdamin ng kabataan? Para sa anong layon o adhikain?

9. Ang literaturang itinuturo ay isang sangay ng kabihasnang kagamitan sa paghulma ng isip at kilos ayon sa dominanteng pangitain ng namumunong uri. Anong pangitain ang gumagana't nanaig?

Indibidwalismong makapamilya o makauri ang istandard, hinaluan ng ilang demokratikong islogan. Inatasan ang guro upang itanim sa utak at puso ang indibidwalismong makasarili kaagapay ng pagsunod sa batas. Hindi bulgar na pangungurakot sapagkat may kaunting aral tungkol sa pakikipagkapwa-tao at pagtulong o pagdamay sa di-kamag-anak, at pagmamahal sa bansa. Sa tradisyonal na ugali, idinidiin ng guro ang aral o ulirang halimbawang mahuhugot sa tula, kuwento, nobela, dula na magsisilbing gabay sa araw-araw na pamumuhay.

10. Sa ganitong pagtingin, ang guro ay gumaganap ng papel ng isang awtoridad, tulad ng pulis o pari. Masunuring estudyante, hindi nagtatanong-ito ba ang hangad natin? Hindi ba kabaligtaran iyon ng nais natin: isang responsableng taong may sariling pangangatwiran? Paano maitatanim at mapapaunlad ang kakayahang mag-isip nang walang alalay, ang maging taong taglay ang kasarinlan at rasong independiyente (autonomous reason) na ideal ng demokrasyang orden, hindi diktadurya o pasistang sosyedad?

11. Ang panitikan ay produkto ng mga kontradiksiyong sosyal at siya ring nagpapaulit-ulit nito. Hindi nasa tinig ng diwa o guniguni ang kahulugan ng akda, kundi nasa pag-antig o pagpukaw ng saloobing nakatugma sa polarisasyon ng mga tauhan at pangyayari. Ang akda ay hindi ekspresyon ng nangungulilang diwa o malay kundi artikulasyon ng senyas o salitang taglay ang magulo't maligalig na ugnayan ng bawat tao sa lipunan.

Sa retorika ng teksto at pagbabalangkas ng naratibo, nakalilikha ng posisyon para sa mga sabjek na kumakatawan sa sistemang nabibiyak, tigib ng nakabibighaning katangian. Ang matingkad na karanasan o reyalidad ay bunga ng diskurso na humuhudyat o tumatawag sa atin upang makisangkot o lumahok sa tunggalian ng mga lakas.

Bumungad sa dalumat ang interogasyon o interpelasyon ng akda. Saan tayo lalagay? Saan tayo papanig, saan makikisanib?

12. Paano susuriin ang ideolohiyang hugis o banghay ng nobela ni Bautista? Sa biglang sipat, mahihinuha na iyon ay nakasalig sa makatotohanang dating at epekto ng tila-realistikong detalye ng paglalarawan, laluna ang eksena ng tortyur, ng maramdaming pagtatalik nina Ana at Roy, atbp. Hindi kailangang magduda na peke o eksaherasyon ang mga pangyayari. Matalas at masinop ang paglalarawan 
ng malagim na karanasan na nakasentro sa mga rebolusyonaryong Ana, Roy, Karla, Jingki, at kanilang pamilya. Ano ang hinihiling sa atin? Ano ang demanda ng nasubaybayang eksena't pangyayari?

Nakaaantig ang pagsisiwalat ng matinding kahirapan ng mga aktibista, ng mabangis na kalupitan ng mga sundalo ng diktaturya, ng matinding galit at kalungkutang sinapit ng mga protagonista. Taglay ng akda ang makasaysayang kakintalang mahirap iwaglit sa kolektibong gunita.

13. Ating ungkatin ang ideolohiya ng teksto sa makinarya ng paghahanay ng mga insidente: Paano nalutas ang problemang gumulo sa buhay ng mga tauhan? Paano nairesolba ang mga kontradiksiyong prinsipal (diktadurya versus demokratikong masa at representatibong partido nito) sa pamamagitan ng imahinaryong paraan, sa pantasya o nais-kaganapan (wish-fulfillment)?

14. Pansining ang suliranin ng kontradiksiyon ng oligarkong pangkat suportado ng imperyalistang Estados Unidos at mga partisano ng demokrasyang pambansa ay naipaloob sa isang kompromisong antas. Ibig sabihin, naisalin iyon sa kuwadro ng problema ng inang nawalan ng anak at sakunang naranasan ng mga aktibistang pinahirapan.

Sa sakripisyo ni Karla, sa kanyang pagmamalasakit, na bunga ng pangingibangbayan (paglipat sa Canada) at pagkalinga ng pamilya, nakuhang ipagtapat sa anak ang katotohanan ng nakalipas. Sumunod naman si Malaya sa paniniwalang ang pagka-ina (maternidad) ay biyolohikal, hindi sosyal; at pagtanggap sa kanya ni Ana bilang simbolo ng kanyang pagsisikap at gantimpala sa pagmamahal sa sarili.

Sa kasukdulan, umabot sa pagkakakilanlan at pagbabalik ng kaginhawahan, bagama't ironikal ang alingawngaw sa dulo. Na wari baga'y may kutob na walang tunay na pagbabagong naganap, may kutob na nasayang ang pawis at dugong ibinuhos ng mga nakipagsapalaran, at bumabalik muli ang sinugpong nakalipas. Ano ba talaga ang kanilang mithiin? Ang katarungan ba'y nakasalalay sa hatol ng hukuman sa Estados Unidos, hindi sa Filipinas? Tila malabo pagdating natin sa dulo.

15. Sa panig ni Roy naman, ang interogasyon ng Komiteng nagsisiyasat tungkol sa mga biktima ng martial-law kaugnay ng kasong ilalapit sa korte sa Estados Unidos ay nagsilbing katarsis upang maibilad niya ang nasugpong yugto ng kanyang buhay, laluna ang pagkitil ng buhay ni Jingki ayon sa atas ng partido, na paghihiganti sa pagkasawi ng buong pamilya niya. Sa tulong ng mga abogadong Filipino at Amerikano, ang katarungan ay natamo, nagkaisa muli ang ina't anak, at nakamit ni Lorie ang kabatiran tungkol sa madugo't lihim na talambuhay ng kanyang magulang. Narekober ang nakalipas, nabuo ang totalidad ng kasaysayan, umabot tayo sa masayang wakas, bagama't may babala na baka maulit muli ang diktadurya sa proklamasyon ni Arroyo sa huling pahina. 
16. Samakatwid, ang tunay na problemang hinarap ng nobela ay nalutas sa imahinaryong paraan, sa paglilipat nito sa isang lunan o palapag na madaling maaayos ang lahat sa paraang pagtawag sa tradisyonal na paniniwala, sukatan o prehuwisyong bumubuo sa lumang kaayusan na siyang pinagmulan ng krisis na sinuong ng mga karakter sa nobela.

Nabuo ang sabjek sa pagtuklas na gumagana pa rin ang tradisyonal na moralidad na batayan ng kilos, isip at damdamin ng tipikal na tauhan sa nobela: pamilya batay sa awtoridad ng magulang, sindak ng militar laban sa rebeldeng pangkat na dapat katakutan, atbp.

Matingkad ang paglalarawan sa problema ng mga anak at magulang, ngunit tila pansamantala lamang ang yugtong iyon, limitado sa ilang tauhan, hindi saklaw ang buong bansa. Lumilitaw na ang diktadurya ay tila anomalya lamang, at ang krisis ay pansamantalang emerhensiya. Ganoon din ang kalabisan ng mga kaliwang puwersa na siyang nagpakilos sa mga aktibista, ngunit hindi sumaklolo o tumulong sa kanilang kahirapan.

Sa namamayagpag na "culture of impunity," tila patuloy ang predikamento ng mga ina. Testigo ang ina ni Jonas Burgos, ang mga magulang nina Karen Empeno at Sherlyn Cadapan, at marami pang iba. Baka maging "desaparecidos" ang buong bansa sa paglabas ng migranteng contract-workers - mahigit 10 milyon na - pati likas-yaman, mga islang lulubog o mawawasak sa bagsik ng napipinsalang kalikasan ng planeta.

Ang pagkawala ng anak ay mistulang sagisag sa pagkawala ng soberanya, kalayaan, integridad ng bansa.

Simbolo kaya ng resureksiyon o muling pagsilang ang pagbabalik ng anak? Sino ang sabjek ng kinabukasang ipinahihiwatig ng pagbuo muli ng pamilya?

17. Kung hindi kayo sang-ayon sa metakomentaryong nailahad, patunay iyon na nangingibabaw pa rin ang tradisyonal na paraan ng pagsusuri't pagpapahalaga sa literatura. Kung nabagabag man kayo, tumalab kahit paano ang proposisyong nailatag dito, maaaring simulang baguhin ang modo ng pagturing sa panitikan bilang repleksiyon ng tinatanggap na realidad o kaya ay ekspresyon ng kaluluwa ng salamangkero ng wika. Simula ring maghuhunos ang pagkilala sa estudyante at guro bilang mga sabjek ng naghaharing paradigma o kwadro ng pagkilatis at pagpapahalaga. Sa gayon ay pwedeng mag-umpisa ang kumbersasyon ng mga komunidad sa impetus ng diyalektikal at materyalismong pagsusuring naibalangkas dito.

18. Sa paglagom, maitanong: taglay ba ng lahat ng akda o diskurso ang hugis ng ideolohiyang nahimay rito? Kung ganoon, walang dapat alalahanin. Walang panganib ang magbasa ng diskursong nag-uulat ng nakaririmarim na mga tagpo, ng mga nakasisindak na pangyayari. Sandaling nagimbal ang kalooban ng mambabasa. 
Sa pag-igkas ng imbestigasyon hinggil sa mga biktima ng diktadurya, sa paginog ng mga insidente, maidadala lahat ng mga masalimuot na kontradiksiyon sa isang lugar na madaling maipapaliwanag ang lahat, at saka maiuugnay sa mapayapa at makabuluhang paraan ang mga nagtutunggaling puwersa upang sa gayon maisauli ang dating status quo at mapatahimik ang lahat. Kung tutuusin, walang dapat ikabahala sa masinop na pag-aaral ng nobelang puno ng madugo at marahas na tagpo.

19. Sa Kanlurang arkibo ng araling kultural, ang genre ng modernong nobela ay nakatuon sa pakikipagsapalaran ng naligaw na kaluluwa sa mundong walang diyos. Ang bayani ay masikhay na naghahanap ng kahulugan o katuturan sa buhay, biktima ng alyenasyon sa daigdig ng komoditi at ipinagbibiling bagay sa mega-mall saanman, napapaligiran ng walang tigil na sigalot, terorismo, digmaan ng mga uri at sari-saring lakas ng nagbubuhat sa gunita ng nakalipas at takot sa malagim na hinaharap.

Sa Filipinas, ang nobela ay nakaugat sa krisis ng pyudal at patriyarkal na orden. Naitampok sa pagsasalaysay ng buhay nina Ana at Roy ang predikamento ng pamilyang nabuwag at mga kabataang napahamak sa pakikibaka sa ngalan ng kalayaan at hustisya, sa kalakaran ng krisis ng neokolonyal na rehimen, kung saan natuklasan ng mambabasa ang tunay na pagkatao ng mga sabjek sa proseso ng pagtataguyod ng rebolusyonaryong balak.

Makapagtuturo kaya ito ng mabuting halimbawa upang ang sabjek na imbensiyon ng diskurso, resulta ng wika ng likhang-sining, ay maitransporma sa mapagpalayang direksiyon? Magagamit kaya ang pormang ideolohikal ng literaturang pinaparangalan ngayon sa makatao at progresibong layunin? Tiyak na ito ay isang kolektibong proyekto na dumudulog sa lahat at humahamon sa ating budhi, puso, katapangan at katapatan. 